\title{
A Research Approximation to Generalized Riemann Derivatives by Integral Operator Families
}

\section{Lutfi Akin}

Department of Business Administration, Faculty of Economics and Administrative Sciences, Mardin Artuklu University, Mardin, Turkey

\section{Email address:}

lutfiakin@artuklu.edu.tr

\section{To cite this article:}

Lutfi Akin. A Research Approximation to Generalized Riemann Derivatives by Integral Operator Families. Mathematics and Computer Science. Vol. 3, No. 1, 2018, pp. 7-12. doi: 10.11648/j.mcs.20180301.12

Received: November 23, 2017; Accepted: December 4, 2017; Published: January 19, 2018

\begin{abstract}
Approximation theory has very important applications of polynomial approximation in various areas of functional analysis, Harmonic analysis, Fourier analysis, application mathematic, operator theory in the field generalized derivatives and numerical solutions of differential and integral equations, etc. Integral operators is very important in Harmonic and Fourier analysis. The study of approximation theory is a well-established area of research which deals with the problem of approximating a function $f$ by means of a sequence $L_{n}$ of positive linear operators. Generalized derivatives (Riemann, Peano and Taylor derivative) are more general than ordinary derivative. Approximation theory is very important for mathematical world. Nowadays, many mathematicians are working in this field.
\end{abstract}

Keywords: Riemann Derivative, Kernel Function, Diferantiable Function, Operator Theory

\section{Introduction}

Integral operators with positive kernel can see in many problems of theory functions and differential equations. For example, some collection methods of the Fourier series are expressed with such integrals. Furthermore, the solution of the Dirichlet problem and the solution of the problem the limit value is given through of positive kernel integrals. Hence, using positive-core integral operators examination the problem of approach to generalized derivatives in terms of both theoretical and practical have great importance (see [1], [2], [3], [4], [6], [7], [8], [9], [10], [11], [12], [13]).

$L_{\lambda}: f(t) \rightarrow L_{\lambda}(f, x)$ integral operators families and $K_{\lambda}(t-x)$ is operator's kernel.

$$
L_{\lambda}(f, x)=\int_{a}^{b} f(t) K_{\lambda}(t-x) d t
$$

The main problems related to the convergence of such integral operator families, it is as follows.

1. Convergence of $L_{\lambda}\left(f, x_{0}\right) \rightarrow f\left(x_{0}\right)$ examining for $\lambda \rightarrow \lambda_{0}$ at a certain $x_{0}$ point.

2. When $X$ is a normative linear space and $f \in X, L_{\lambda}: X \rightarrow X$

$$
\lim _{\lambda \rightarrow \lambda_{0}}\left\|L_{\lambda} f-f\right\|_{X}=0
$$

3. Finding the convergence speeds of problems 1 and 2 , that is, when $\lambda \rightarrow \lambda_{0}$ and $\alpha_{\lambda}, \beta_{\lambda}$ are zero series

$$
\begin{aligned}
& \left|L_{\lambda}\left(f, x_{0}\right)-f\left(x_{0}\right)\right|=o\left(\alpha_{\lambda}\right), \\
& \left\|L_{\lambda} f-f\right\|_{X}=o\left(\beta_{\lambda}\right), \\
& \left|L_{\lambda}\left(f, x_{0}\right)-f\left(x_{0}\right)\right|=O\left(\alpha_{\lambda}\right), \\
& \left\|L_{\lambda} f-f\right\|_{X}=O\left(\beta_{\lambda}\right),
\end{aligned}
$$

proof of such proposals,

4. Problem finding of asymptotic value of approach is more than the problem finding of approach speed. Asymptotic value approach to $f$ function at a certain $x_{0}$ point of family integral operators $L_{\lambda}$ examined by mathematicians. Convolute type such problems are studied by Weierstrasse, Gauss, Perron, Landau, Picard, Lebesgue, Faddeev, Romanovsky, Natanson, Korovkin, Butzer. 


\section{Auxillary Statements and Definitions}

Theorem 2.1. [2], [13] If $f(x)$ function there is left and right derivative at a certain point $x_{0}$ then,

$$
\lim _{n \rightarrow \infty} \frac{F_{n}\left(f ; x_{0}\right)-f\left(x_{0}\right)}{F_{n}\left(\left|\sin \frac{t}{2}\right| ; 0\right)}=f_{+}^{\prime}\left(x_{0}\right)-f_{-}^{\prime}\left(x_{0}\right)
$$

equality is hold.

$$
\begin{aligned}
& \text { Proof. Let, } \lim _{t \rightarrow 0^{+}} \frac{f\left(t+x_{0}\right)-f\left(x_{0}\right)}{t}=f_{+}^{\prime}\left(x_{0}\right) \text { and } \\
& \lim _{t \rightarrow 0^{-}} \frac{f\left(t+x_{0}\right)-f\left(x_{0}\right)}{t}=f_{-}^{\prime}\left(x_{0}\right) \\
& \phi(t)=\frac{f\left(t+x_{0}\right)-f\left(x_{0}-t\right)}{2}-f\left(x_{0}\right) \text { and } \psi(t)=\frac{f\left(t+x_{0}\right)+f\left(x_{0}-t\right)}{2}
\end{aligned}
$$

From here,

$$
\phi(t)+\psi(t)=f\left(t+x_{0}\right)-f\left(x_{0}\right)
$$

is obtained. Now,

$$
\lim _{t \rightarrow 0} \frac{\phi(t)}{\left|\sin \frac{t}{2}\right|}=f_{+}^{\prime}\left(x_{0}\right)-f_{-}^{\prime}\left(x_{0}\right)
$$

For $t \rightarrow 0^{-}$.

$$
\begin{aligned}
& \lim _{t \rightarrow 0^{-}} \frac{\phi(t)}{\left|\sin \frac{t}{2}\right|}= \lim _{t \rightarrow 0^{-}} \frac{\phi(t)}{-\sin \frac{t}{2}}=\lim _{t \rightarrow 0^{-}} \frac{f\left(t+x_{0}\right)-f\left(x_{0}\right)}{t} \cdot \frac{t}{-\sin \frac{t}{2}}+ \\
&+\frac{1}{2} \lim _{t \rightarrow 0^{-}} \frac{f\left(t+x_{0}\right)-f\left(x_{0}\right)}{-t} \cdot \frac{t}{\sin \frac{t}{2}} \\
&=\frac{1}{2}\left\{f_{-}^{\prime}\left(x_{0}\right) \cdot(-2)+f_{+}^{\prime}\left(x_{0}\right) \cdot 2\right\}=f_{+}^{\prime}\left(x_{0}\right)-f_{-}^{\prime}\left(x_{0}\right)
\end{aligned}
$$

is hold. For $t \rightarrow 0^{+}$

$$
\begin{gathered}
F_{n}\left(f ; x_{0}\right)-f\left(x_{0}\right)=\frac{1}{n \pi} \int_{-\pi}^{\pi}\left\{f\left(x_{0}+t\right)-f\left(x_{0}\right)\right\} \frac{\sin ^{2}\left(\frac{n t}{2}\right)}{2 \sin ^{2}\left(\frac{t}{2}\right)} d t \\
=\frac{1}{n \pi} \int_{-\pi}^{\pi} \phi(t) \frac{\sin ^{2}\left(\frac{n t}{2}\right)}{2 \sin ^{2}\left(\frac{t}{2}\right)} d t+\frac{1}{n \pi} \int_{-\pi}^{\pi} \psi(t) \frac{\sin ^{2}\left(\frac{n t}{2}\right)}{2 \sin ^{2}\left(\frac{t}{2}\right)} d t
\end{gathered}
$$

is obtained. This last integral is equal to zero. Therefore,

$$
F_{n}\left(f ; x_{0}\right)-f\left(x_{0}\right)=\frac{1}{n \pi} \int_{-\pi}^{\pi} \phi(t) \frac{\sin ^{2}\left(\frac{n t}{2}\right)}{2 \sin ^{2}\left(\frac{t}{2}\right)} d t=F_{n}(\phi ; 0)
$$

is obtained. From here,

$$
\begin{gathered}
\lim _{n \rightarrow \infty} \frac{F_{n}\left(f ; x_{0}\right)-f\left(x_{0}\right)}{F_{n}\left(\left|\sin \frac{t}{2}\right| ; 0\right)}=\lim _{n \rightarrow \infty} \frac{F_{n}(\phi ; 0)}{F_{n}\left(\left|\sin \frac{t}{2}\right| ; 0\right)}=\lim _{t \rightarrow 0} \frac{\phi(t)}{\left|\sin \frac{t}{2}\right|}= \\
f_{+}^{\prime}\left(x_{0}\right)-f_{-}^{\prime}\left(x_{0}\right)
\end{gathered}
$$

is obtained. Hence,

$$
\begin{gathered}
\lim _{n \rightarrow \infty} \frac{F_{n}\left(f ; x_{0}\right)-f\left(x_{0}\right)}{F_{n}\left(\left|\sin \frac{t}{2}\right| ; 0\right)}=\lim _{n \rightarrow \infty} \frac{F_{n}(\phi ; 0)}{F_{n}\left(\left|\sin \frac{t}{2}\right| ; 0\right)}=\lim _{t \rightarrow 0} \frac{\phi(t)}{\left|\sin \frac{t}{2}\right|}= \\
f_{+}^{\prime}\left(x_{0}\right)-f_{-}^{\prime}\left(x_{0}\right)
\end{gathered}
$$

is obtained. This proof is completed.

Theorem 2.2. If sequence linear positive operators $L_{n}$ satisfies the following conditions in $[a, b]$. This symbol $\rightarrow$ is meaning of aproximation, for example, $1 \rightarrow 1, t \rightarrow x$, $\left.t^{2} \rightarrow x^{2}\right)$

$$
\begin{aligned}
L_{n}(1 ; x) & \rightarrow 1 \\
& \rightarrow \\
L_{n}(t ; x) & \rightarrow x \\
& \rightarrow x^{2} \\
L_{n}\left(t^{2} ; x\right) & \rightarrow
\end{aligned}
$$

In this case, when $\mathrm{n}$ goes infinitely for any $f$ function in space $C(a, b)$,

$$
L_{n}(f ; x) \underset{f(x), a \leq x \leq b}{\rightarrow}
$$

condition is satisfied (see [2], [13])

Theorem 2.3. Let $f(x)$ function differentiable from the $(n-1)$ th order neighbourhood at a certain point $x_{0}$ and let $f(x)$ function has left and right derivative from $n$th order at a certain point $x_{0}, f_{+}^{(n)}\left(x_{0}\right), f_{-}^{(n)}\left(x_{0}\right) .1 \leq \phi(x)<\infty$ for $x \in(-\infty, \infty), \quad|f(x)| \leq \phi(x)$ inequality is provide. $K_{\lambda}(t)$ is non-negative and even function. At the same time

$$
\int_{-\infty}^{\infty} K_{\lambda}(t) d t=1
$$

Let for $\forall \delta>0$,

$$
\mu(t)=\sup _{\substack{-\infty<x<\infty \\|y|<t}} \frac{\phi(x+y)}{\phi(x)}<\infty
$$


while $\lambda \rightarrow \infty$ if the following equality is satisfied

$$
\int_{\delta}^{\infty} \mu\left(\alpha^{*} t\right) \Phi(t) K_{\lambda}(t) d t=o\left(\Delta_{\lambda}\right)
$$

then,

$$
\lim _{\lambda \rightarrow+\infty} \frac{L_{\lambda}\left(f ; x_{0}\right)-f\left(x_{0}\right)}{R_{n, \lambda} \cdot \Delta_{\lambda}}=\frac{f_{+}^{n}\left(x_{0}\right) \pm f_{-}^{n}\left(x_{0}\right)}{n !}
$$

equality is provided (see [3], [4], [13]).

Definition 2.4. $f$ is defined in some neighbourhood of the point $x_{0}$ and if the $(r-1)$ th ordinary derivative $f^{r-1}\left(x_{0}\right)$ exists, then we call

$$
f^{(r)}\left(x_{0}\right)=\lim _{h \rightarrow 0} \frac{r !}{h^{r}}\left[f\left(x_{0}+h\right)-\sum_{k=0}^{r-1} \frac{h^{k}}{k !} f^{k}\left(x_{0}\right)\right]
$$

the $r$ th Taylor derivative of $f$ at $x_{0}$ if the limit exists (see [5]).

Lemma 2.5. If the first-order ordinary derivative $f^{\prime}(x)$ exists, so does the first-order Taylor derivative $f^{(1)}(x)$, and we have $f^{\prime}(x)=f^{(1)}(x)$. At the same time, the opposite of this lemma is true (see [5]).

Proof. If $f(x)$ function there is a first-order derivative at point $x_{0}$,

$$
f^{\prime}\left(x_{0}\right)=\lim _{h \rightarrow 0} \frac{f\left(x_{0}+h\right)-f\left(x_{0}\right)}{h}
$$

this derivative is also the first-order Taylor derivative

$$
f^{(1)}\left(x_{0}\right)=\lim _{h \rightarrow 0} \frac{f\left(x_{0}+h\right)-f\left(x_{0}\right)}{h}
$$

Therefore,

$$
f^{\prime}\left(x_{0}\right)=f^{(1)}\left(x_{0}\right)
$$

Lemma 2.6. If the second-order ordinary derivative $f^{\prime \prime}(x)$ exists, so does the second-order Taylor derivative $f^{(2)}(x)$, and we have $f^{\prime \prime}(x)=f^{(2)}(x) . f(x)$ has second-order Taylor derivative at $x=x_{0}$, but there exists no second-order ordinary derivative $x=x_{0}$ (see [5]).

Proof. Taylor expansion at $x_{0}$ point of $f(x)$ function is below

$$
f(x)=f\left(x_{0}\right)+\frac{f^{\prime}\left(x_{0}\right)}{1 !}\left(x-x_{0}\right)+\frac{f^{\prime \prime}\left(x_{0}\right)}{2 !}\left(x-x_{0}\right)^{2}+\ldots \ldots .
$$

If $x$ is replaced by $x_{0}+h$

$$
\begin{gathered}
f\left(x_{0}+h\right)=f\left(x_{0}\right)+\frac{f^{\prime}\left(x_{0}\right)}{1 !} h+\frac{\left(f^{\prime \prime}\left(x_{0}\right)+\alpha\right)}{2 !} h^{2} \quad h \rightarrow 0, \\
\alpha \rightarrow 0
\end{gathered}
$$

is obtained. From here,

$$
\begin{gathered}
f\left(x_{0}+h\right)-f\left(x_{0}\right)-f^{\prime}\left(x_{0}\right) h=\frac{\left(f^{\prime \prime}\left(x_{0}\right)+\alpha\right)}{2 !} h^{2} \\
\frac{2}{h^{2}}\left[f\left(x_{0}+h\right)-f\left(x_{0}\right)-f^{\prime}\left(x_{0}\right) h\right]=\lim _{h \rightarrow 0}\left(f^{\prime \prime}\left(x_{0}\right)+\alpha\right)
\end{gathered}
$$

since,

$$
f^{(2)}\left(x_{0}\right)=f^{\prime \prime}\left(x_{0}\right)
$$

is obtained ( $f(x)$ has second-order Taylor derivative at $x=x_{0}$, but there exists no second-order ordinary derivative $x=x_{0}$, for example see [5]

Theorem 2.7. [13] Let $f(x)$ function differentiable from the $(n-1)$ th order neighbourhood at a certain point $x_{0}$. And let $f(x)$ function has derivative from $n$th order at a certain point $x_{0}$ meaning of Taylor.

And let integral operator families,

$$
L_{\lambda}\left(f ; x_{0}\right)=\int_{-\infty}^{\infty} f\left(x_{0}+t\right) K_{\lambda}(t) d t .
$$

$$
\Delta_{\lambda}=\int_{0}^{\infty} t^{2} K_{\lambda}(t) d t \rightarrow 0 \quad, \quad(\lambda \rightarrow \infty)
$$

For $K_{\lambda}(t)$ kernel function,
i. $K_{\lambda}(t)>0, \quad \lambda \geq 0$
ii. $K_{\lambda}(-t)=K_{\lambda}(t)$
iii. $\int_{-\infty}^{\infty} K_{\lambda}(t) d t=1$

$$
\begin{gathered}
\int_{-\infty}^{\infty} K_{\lambda}(t) d t=2 \int_{0}^{\infty} K_{\lambda}(t) d t \\
\lim _{\lambda \rightarrow \infty} \frac{L_{\lambda}\left(f, x_{0}\right)-f\left(x_{0}\right)}{\Delta_{\lambda}}=f^{(2)}\left(x_{0}\right)
\end{gathered}
$$

equality is provide.

Proof. Let

$$
\begin{aligned}
& L_{\lambda}\left(f, x_{0}\right)-f\left(x_{0}\right) \\
& =\int_{0}^{\infty}\left[f\left(x_{0}+t\right)-2 f\left(x_{0}\right)\right] K_{\lambda}(t) d t=
\end{aligned}
$$




$$
\begin{array}{r}
=\int_{0}^{\infty}\left[f\left(x_{0}+t\right)-f\left(x_{0}\right)-t f^{\prime}\left(x_{0}\right)+t f^{\prime}\left(x_{0}\right)\right] K_{\lambda}(t) d t \\
-\int_{0}^{\infty} f\left(x_{0}\right) K_{\lambda}(t) d t=\int_{0}^{\infty}\left[f\left(x_{0}+t\right)-f\left(x_{0}\right)-t f^{\prime}\left(x_{0}\right)\right] K_{\lambda}(t) d t+ \\
\int_{0}^{\infty} t f^{\prime}\left(x_{0}\right) K_{\lambda}(t) d t-\int_{0}^{\infty} f\left(x_{0}\right) K_{\lambda}(t) d t
\end{array}
$$

Now, define a function as follows

$$
\alpha_{\lambda}(t)=\left[f\left(x_{0}+t\right)-f\left(x_{0}\right)-t f^{\prime}\left(x_{0}\right)\right]-t^{2} f^{(2)}\left(x_{0}\right)
$$

From here, $\alpha_{\lambda}(t) \rightarrow 0$ while $t \rightarrow 0$

$$
\left[f\left(x_{0}+t\right)-f\left(x_{0}\right)-t f^{\prime}\left(x_{0}\right)\right]=\alpha_{\lambda}(t)+t^{2} f^{(2)}\left(x_{0}\right)
$$

is obtained. If $f\left(x_{0}+t\right)-f\left(x_{0}\right)-t f^{\prime}\left(x_{0}\right)$ is replaced by $\alpha_{\lambda}(t)+t^{2} f^{(2)}\left(x_{0}\right)$ above (7)

$$
\begin{gathered}
L_{\lambda}\left(f, x_{0}\right)-f\left(x_{0}\right)=\int_{0}^{\infty} \alpha_{\lambda}(t) K_{\lambda}(t) d t+f^{(2)}\left(x_{0}\right) \int_{0}^{\infty} t^{2} K_{\lambda}(t) d t+ \\
+f^{\prime}\left(x_{0}\right) \int_{0}^{\infty} t K_{\lambda}(t) d t-f\left(x_{0}\right) \int_{0}^{\infty} K_{\lambda}(t) d t
\end{gathered}
$$

is obtained. Thus the proof is completed.

Definition 2.8. [5] $f$ function is defined in a neighbourhood of the point $x_{0}$, then we call

$$
f^{[n]}\left(x_{0}\right)=\lim _{h \rightarrow 0} \frac{1}{h^{n}}\left[\sum_{k=0}^{n}(-1)^{k}(n, k) f\left(x_{0}+\left(\frac{n}{2}-k\right) h\right)\right]
$$

the $n$th Riemann derivative of $f$ at $x_{0}$ if the limit exists and finite.

Lemma 2.9. [5] If the first-order ordinary derivative $f^{\prime}(x)$ exists, so does the first-order Riemann derivative $f^{[1]}(x)$, and we have $f^{\prime}(x)=f^{[1]}(x)$. At the same time, the opposite of this lemma is true.

Proof. If $f(x)$ function there is a first-order derivative at point $x_{0}$,

$$
f^{\prime}\left(x_{0}\right)=\lim _{h \rightarrow 0} \frac{f\left(x_{0}+h\right)-f\left(x_{0}\right)}{h}
$$

this derivative is also the first-order Riemann derivative

$$
f^{[1]}\left(x_{0}\right)=\lim _{h \rightarrow 0} \frac{f\left(x_{0}+h\right)-f\left(x_{0}\right)}{h}
$$

Taylor expansion at $x_{0}$ point of $f(x)$ function is below

$$
f(x)=f\left(x_{0}\right)+\frac{f^{\prime}\left(x_{0}\right)}{1 !}\left(x-x_{0}\right)+\frac{f^{\prime \prime}\left(x_{0}\right)}{2 !}\left(x-x_{0}\right)^{2}+\ldots \ldots
$$

If $x$ is respectively replaced by $x_{0}+\frac{h}{2}$ and $x_{0}-\frac{h}{2}$

$$
\begin{aligned}
& f\left(x_{0}+\frac{h}{2}\right)=f\left(x_{0}\right)+\frac{\left(f^{\prime}\left(x_{0}\right)+\beta_{1}\right)}{1 !} \frac{h}{2}, \beta_{1} \rightarrow 0 \\
& f\left(x_{0}-\frac{h}{2}\right)=f\left(x_{0}\right)-\frac{\left(f^{\prime}\left(x_{0}\right)+\beta_{2}\right)}{1 !} \frac{h}{2}, \beta_{2} \rightarrow 0
\end{aligned}
$$

is obtained. From here,

$$
f\left(x_{0}+\frac{h}{2}\right)-f\left(x_{0}-\frac{h}{2}\right)=\left[f^{\prime}\left(x_{0}\right)+\left(\beta_{1}+\beta_{2}\right)\right] h
$$

is obtained. For $\beta_{1}+\beta_{2}=\beta$

$$
f^{\prime}\left(x_{0}\right)+\beta=\frac{1}{h}\left[f\left(x_{0}+\frac{h}{2}\right)-f\left(x_{0}-\frac{h}{2}\right)\right]
$$

is obtained. From limit definition and $h \rightarrow 0, \beta \rightarrow 0$,

$$
\lim _{h \rightarrow 0}\left(f^{\prime}\left(x_{0}\right)+\beta\right)=\lim _{h \rightarrow 0} \frac{1}{h}\left[f\left(x_{0}+\frac{h}{2}\right)-f\left(x_{0}-\frac{h}{2}\right)\right]=f^{[1]}\left(x_{0}\right)
$$

is obtained.

Lemma 2.10. [5] If the second-order ordinary derivative $f^{\prime \prime}(x)$ exists, so does the second-order Riemann derivative $f^{[2]}(x)$ and we have $f^{\prime \prime}(x)=f^{[2]}(x) \cdot f(x)$ has second-order Riemann derivative at $x=x_{0}$ but there exists no second-order ordinary derivative $x=x_{0}$. (for example see [5])

Proof. Taylor expansion at $x_{0}$ point of $f(x)$ function is below

$$
f(x)=f\left(x_{0}\right)+\frac{f^{\prime}\left(x_{0}\right)}{1 !}\left(x-x_{0}\right)+\frac{f^{\prime \prime}\left(x_{0}\right)}{2 !}\left(x-x_{0}\right)^{2}+\ldots \ldots
$$

If $x$ is respectively replaced by $x_{0}+h$ and $x_{0}-h$

$$
\begin{aligned}
& f\left(x_{0}+h\right)=f\left(x_{0}\right)+\frac{f^{\prime}\left(x_{0}\right)}{1 !} h+\frac{\left(f^{\prime \prime}\left(x_{0}\right)+\beta_{1}\right)}{2 !} h^{2},\left(\beta_{1} \rightarrow 0\right) \text { and } \\
& f\left(x_{0}-h\right)=f\left(x_{0}\right)-\frac{f^{\prime}\left(x_{0}\right)}{1 !} h+\frac{\left(f^{\prime \prime}\left(x_{0}\right)+\beta_{2}\right)}{2 !} h^{2},\left(\beta_{2} \rightarrow 0\right)
\end{aligned}
$$

is obtained. From here

$$
\begin{gathered}
f\left(x_{0}+h\right)+f\left(x_{0}-h\right)=2 f\left(x_{0}\right)+\left(f^{\prime \prime}\left(x_{0}\right)+\frac{\beta_{1}+\beta_{2}}{2}\right) h^{2}, \\
\left(\frac{\beta_{1}+\beta_{2}}{2}=\beta \rightarrow 0\right)
\end{gathered}
$$$$
\frac{1}{h^{2}}\left[f\left(x_{0}+h\right)-2 f\left(x_{0}\right)+f\left(x_{0}-h\right)\right]=\left(f^{\prime \prime}\left(x_{0}\right)+\beta\right)
$$ 
is obtained. From limit definition and $h \rightarrow 0, \beta \rightarrow 0$,

$$
\lim _{h \rightarrow 0} \frac{1}{h^{2}}\left[f\left(x_{0}+h\right)-2 f\left(x_{0}\right)+f\left(x_{0}-h\right)\right]=\lim _{h \rightarrow 0}\left(f^{\prime \prime}\left(x_{0}\right)+\beta\right)
$$

is hold.

From here $f^{[2]}\left(x_{0}\right)=f^{\prime \prime}\left(x_{0}\right)$ is hold. ( $f(x)$ has secondorder Riemann derivative at $x=x_{0}$, but there exists no second-order ordinary derivative $\left.x=x_{0}\right)$.

Example 2.11. [5] For $f(x)= \begin{cases}x \sin \left(x^{-2}\right) & x \neq 0 \\ 0 & x=0\end{cases}$

$f(x)$ has second-order Riemann derivative at $x=0$, but there exists no second-order ordinary derivative $x=0$.

\section{Main Result}

Theorem 3.1. Let $f(x)$ function differentiable from the second-order ordinary derivative neighbourhood at a certain point $x_{0}$. and let integral operator families,

$$
\begin{aligned}
& L_{\lambda}\left(f ; x_{0}\right)=\int_{-\infty}^{\infty} f\left(x_{0}+t\right) K_{\lambda}(t) d t \\
& \Delta_{\lambda}=\int_{0}^{\infty} t^{2} K_{\lambda}(t) d t \rightarrow 0,(\lambda \rightarrow \infty)
\end{aligned}
$$

For $K_{\lambda}(t)$ kernel function,

$$
\begin{aligned}
& \text { i. } K_{\lambda}(t)>0, \lambda \geq 0 \\
& \text { ii. } K_{\lambda}(-t)=K_{\lambda}(t) \\
& \text { iii. } \int_{-\infty}^{\infty} K_{\lambda}(t) d t=1, \int_{-\infty}^{\infty} K_{\lambda}(t) d t=2 \int_{0}^{\infty} K_{\lambda}(t) d t \\
& \qquad \lim _{\lambda \rightarrow \infty} \frac{L_{\lambda}\left(f, x_{0}\right)-f\left(x_{0}\right)}{\Delta_{\lambda}}=f^{[2]}\left(x_{0}\right)
\end{aligned}
$$

equality is hold.

Proof. Let

$$
L_{\lambda}\left(f, x_{0}\right)-f\left(x_{0}\right)=\int_{0}^{\infty}\left[f\left(x_{0}+t\right)-2 f\left(x_{0}\right)+f\left(x_{0}-t\right)\right] K_{\lambda}(t) d t
$$

Now, let define a function as follows for $\Lambda_{\lambda}(t) \rightarrow 0$ while $t \rightarrow 0$

$$
\Lambda_{\lambda}(t)=\left[f\left(x_{0}+t\right)-2 f\left(x_{0}\right)+f\left(x_{0}-t\right)\right]-t^{2} f^{[2]}\left(x_{0}\right)
$$

From here,

$$
\left[f\left(x_{0}+t\right)-2 f\left(x_{0}\right)+f\left(x_{0}-t\right)\right]=\Lambda_{\lambda}(t)+t^{2} f^{[2]}\left(x_{0}\right)
$$

is obtained.

If $\left[f\left(x_{0}+t\right)-2 f\left(x_{0}\right)+f\left(x_{0}-t\right)\right]$ is replaced by $\Lambda_{\lambda}(t)+t^{2} f^{[2]}\left(x_{0}\right)$ above $(9)$

$$
\begin{gathered}
L_{\lambda}\left(f, x_{0}\right)-f\left(x_{0}\right)=\int_{0}^{\infty}\left[\Lambda_{\lambda}(t)+f^{[2]}\left(x_{0}\right) t^{2}\right] \cdot K_{\lambda}(t) d t \\
=\int_{0}^{\infty} \Lambda_{\lambda}(t) K_{\lambda}(t) d t+f^{[2]}\left(x_{0}\right) \int_{0}^{\infty} t^{2} K_{\lambda}(t) d t
\end{gathered}
$$

is obtained. Thus the proof is completed.

\section{Conclusion}

According to research approximation to Riemann derivative with integral operator families, Riemann derivative is more general than ordinary derivative.

Example 4.1. For $f(x)=|x|$, This function has Riemann derivative at the point $x=0$. But this function has not ordinary derivative at the point $x=0$.

\section{Acknowledgements}

The author expressed their gratitude to the referee for helpful remarks. And this work is supported by MAU-BAP17-IIBF-07

\section{References}

[1] Altomare, F. and Campiti, M. 1994. Korovkin Type Approximation Theory and its Application, Walter de Gruyter, Berlin and NewYork.

[2] Korovkin, P. P., 1960. Linear Positive Operators and Approximation Theory. Hindistan Publishing Corp., 222pp, Hindustan.

[3] Gadjiev, A. D., Djifarov, A. S. and Labsker, L. G., 1962. On Asymtotic Value of Approximation of Functions By Certain Families of Integral Operators, Izvestiya Acad. Sci. of The Azerbaijan., 3: 19-28. In Russian.

[4] Gadjiev, A. D., 1962. On Asymptotic Values of Approximation of Derivatives of functions By Derivatives of Families of Linear Operators. Izvestiya Acad. of Sci. of Azebaijan, 6:15-24.

[5] Butzer, P. L. and Nessel, R. J., 1971. Fourier Analysis and Approximation, Academic Pres, 555p, New York and London.

[6] Akin, L., 2008. "Approximation to Generalized Derivatives by Integral Operator Families.”, Harran University, Institute of Science, Master Thesis, Sanliurfa, 55p.

[7] Butzer, P. L., 1960. Representation and Approximation of Function By General Singular Integrals, Proceeding Konikel. Acad. Wet 63, Nederland.

[8] Hacisalihoğlu, H. and Haciyev, A., 1995. "Convergence of Linear Positive Operator Sequences.”, Ankara, 100s, Ankara.

[9] Stein, E. M., 1993. Singular Integrals and Differentiability 
Properties of Functions, Princeton Univ. Pres., 289pp, The United States of America.

[10] Zeren, Y., 2006. Approximation of Schwartz Differentiable Functions of Several Variables by the Sequence of Integral Operators. Khazar Journal of mathematics, 1:73-78.

[11] Zeren, Y., 2002. "Asymptotic Value of Approximation of Derivatives Approximated by Integral Operator Family.", Ankara University, Institute of Science, Ph. D. Tesis Ankara,
$50 \mathrm{p}$.

[12] Mamedov I. F., Zeren Y., Akın L., 2017. "Compactification of Weighted Hardy Operator in Variable Exponent Lebesgue Spaces", Asian Journal Mathematics and Computer Research, $17(1): 38-47$.

[13] Akin L. and Zeren Y., 2017. "Approximation to generalized Taylor derivative by integral operator families", MSU J. of Sci., Volume 5, Issue 2, p 421-423. 